\title{
Review of Machine Vision Based Insulator Inspection Systems for Overhead Power Distribution System
}

\author{
P. Surya Prasad ${ }^{1}$, B. Prabhakara Rao ${ }^{2}$ \\ ${ }^{1}$ Department of ECE, MVGR College of Engineering, Vizianagaram, Andhra Pradesh, India \\ ${ }^{2}$ Department of ECE, JNTU Kakinada, Andhra Pradesh, India
}

\begin{tabular}{l} 
Article Info \\
\hline Article history: \\
Received Sep 13, 2017 \\
Revised Nov 13, 2017 \\
Accepted Nov 21, 2017 \\
\hline
\end{tabular}

Keyword:

Classification

Condition monitoring

Defect detection

Feature extraction

Image processing

Machine learning

\begin{abstract}
The necessity to have reliable and quality power distribution is increasing, and hence there is great scope for research on automation of distribution system. There are signs of increased research in the work on condition monitoring of insulators during the last few decades. The possible failures can be predicted before they actually occur by using the condition monitoring of cables or any electrical equipment on-line. Those assets such as towers, conductors and insulators which are on the threshold of failure have to be replaced or repaired, so that forced outages reduce. Traditionally the workers who inspect these lines check them in close proximity by going for footpatrolling and pole-climbing. With an incredible expansion of power distribution network even to remote areas, previously mentioned methods do not seem to be viable. In developed countries aerial patrolling has been adopted to monitor the insulators as an alternative. The development of an efficient method of condition monitoring by using image processing followed by machine learning techniques is found to be a suitable method and thus emerging as a feasible option for real-time implementation. This review paper covers overall aspects of automatic detection of defects of insulator systems of electric power lines and classification into different classes by using vision-based techniques.
\end{abstract}

Copyright @ 2017 Institute of Advanced Engineering and Science. All rights reserved.

Corresponding Author:

P. Surya Prasad,

Department of ECE, MVGR College of Engineering,

Vizianagaram, Andhra Pradesh, India.

Email: suryaprasadp@yahoo.com

\section{INTRODUCTION}

Throughout worldwide electric power utilities are adopting the computer aided monitoring, management and control of electric power distribution system more and more, to provide better services to the consumers of electricity [1]. The idea of distribution automation began in 1970s and the motivation to improve operating performance of distribution systems was derived from the usage of computer and communications technology. Then onwards, the improvement in automation of distribution sestym has been dictated by increasing complexity levels in the existing technologies for monitoring, control, communication technologies and also the performance and cost of the equipment [24]. To ensure uninterrupted reliable operation of power distribution system, the performance of insulators under different environmental conditions must be reliable and satisfactory. So, the supplier has to take all the necessary steps to inspect his installations and ensure that their performance comply with the regulations. The other reasons to perform inspections of lines regularly are: i. Detection of line defects early reduces power cuts and it paves the way for good customer care and advantage over other competitors; ii. Having a photographic record allows the repair teams to know exactly about the repair and maintenance to be done which reduces operational costs; iii. Public are protected by detecting the missing or broken safety features on poles [2]. If a device is found to 
be the cause of the fault, repair or replacement of the same could be made or recommendations are given for any other required maintenance actions [3].

If the distribution equipment starts weakening, it can be anticipated that an unpredictable fault is developing in the system may be from several days or several months. So, in order to have a reliable power distribution system, it is crucial to monitor distribution, classify and identify the various types of failures before the actual breakdown or breakout of the equipment occurs [3]. The on-line processing to monitor the health of equipment is generally called as condition monitoring (CM) [4]. The overhead lines have many items such as insulators, conductors and fittings, which can be continuously monitored online [5-11]. So, there should be a constant monitoring of the system to trace its progression from healthy to sick status. A mention about health index based on monitoring data is presented in [12]. So, the development of transducer technologies, computer technologies and signal processing techniques along with artificial-intelligence (AI) techniques has made it possible to implement CM more effectively on electrical equipment [13]. This review aims at comparing and contrasting the reported research that was done based on Image Processing along with Machine Learning Techniques in computing some meaningful information obtained from condition monitoring.

\section{COMPLEXITIES OF AUTOMATION OF INSULATOR MONITORING}

Accurate and reliable techniques to locate, detect failures, identification and evaluation of severity, have become crucial to in dealing with the maintenance work in the scheduled time and realistic cost [4]. On-site Manual detection is costly, time consuming, and is an impractical task in case of monitoring the long lines spread over a large distance and difficult terrains. Video surveillance system based on image processing can do that type of monitoring very easily. Use of helicopters has been proposed for video surveillance in UK to inspect the distribution system. Jones and Earp [15] gave detailed discussion on the motivation to go for video inspection techniques and the problems that may arise. And there is a mention of wide application of aerial inspection [16] for condition monitoring of overhead lines.

Automatic video surveillance using a helicopter of power lines is not as straightforward and the problems are: a. Pattern recognition applied to target locations [17], b. Stabilization the camera in compensation of the helicopter's 6 degree-of-freedom (DOF) movement [18,19], c. Acquire and maintain the target in the camera's field of view (FOV) [17], iv) Camera's residual sightline motion results image degradation $[18,15,20]$, and v) Data analysis system. The inspection process becomes automatic by the use of video surveillance techniques and it also improves its depth and coverage because it provides permanent record of the images [14]. But, the inspection of insulators in real-time faces a number of challenges as follows: Image blurring, camera sight control, fast changing background, and gradual intrusion of tree limbs into the overhead power lines [9]. As an improvement, video surveillance with fixed cameras as applied for pedestrian detection [21] and this seems to be a promising solution for video surveillance of power distribution system insulators. With the fast changing scenario in availability of cheaper digital cameras with good performance, the mounting of video cameras became cheap. But the man power becomes very expensive to personally observe and interpret the results. So it was proposed to operate cameras at regular intervals of time to get images of power distribution lines along with insulators, sent for analysis to the control room by the use of remote terminal unit (RTU)s [9] and this method assures promising results.

\section{PRIOR LITERATURE REVIEW}

In the last few decades, there has been a lot of research conducted in the field of automatic power line inspection. A review paper, addressing exclusively the inspection with aerial vehicles is given in [25]. A survey paper has been published about the overhead power line inspection which includes automated helicopter-assisted inspection based on inspection with flying and climbing robots [27]. Over the years, there are a number of review papers on condition monitoring of distribution power line inspection $[2-4,23-27,32,38]$.

A presentation on feasibility study about characterization of emerging insulator failure to predict fault in the distribution is given in [2]. A brief outline on overhead line deterioration, available inspection methods and information about a project being undertaken by the Power and Energy Systems Research Group at the University of Bath who monitored overhead lines on-line [4]. The development of a new twocourse sequence to reflect the radical changes that occurs or expected to happen in future was recommended by the author in [23]. An automatic video surveillance system using a manned helicopter was proposed to be a promising alternative for traditional inspection methods of power lines in [25] and remotely operated unmanned flying robot was anticipated as the future of overhead power line inspection. B.Avidar in [26] gives brief explanation of the inspection methods in works and focuses on the electronic approach. He also 
discusses the concept of a airborne, completely stand and electronic method [26]. The most prominent achievements about inspection of power distribution line by mobile robots are presented in [27]. The insufficiency of traditional ways of insulator detection has headed towards lot of research on automatic online detection method. Among the various methods of detection surveyed in [38], the electric field distribution method detects the internal insulation defects live line. An excellent theoretical background to image processing is covered by Gonzalez and Woods [30] and classification based on neural network is given Haykins [31].

Availability of the published literature on automated monitoring of insulators' condition mainly consists of research work done at several academic and research institutions. Academic institutes have published number of research works. There are some papers published in reported research work mainly focuses on classification of defects of insulators of power distribution, implemented using various kinds of approaches. However, the authors could not find any review paper on the research work done in the field of condition monitoring and classification of insulators of distribution system using image processing combined with artificial machine learning techniques. Therefore, this attempt is being done to consolidate the published literature from academia and research institutes on the topic of automatic inspection of the condition of insulators. It then throws a light on various detection and analysis techniques presently being used. The aim of this paper is to compare and contrast the analyses aimed to detect and classify the defected or cracked insulators and thus contribute to the design and deployment of an on-line condition monitoring through the use of machine vision techniques [4].

\section{CATEGORIES OF INSULATORS AND DEFECTS}

The insulators used in transmission line are the devices which are used to contain, support or separate the electrical conductors. They are used for high voltage power distribution networks. The transmission insulators are available in various types and shapes, which include individual or strings of disks, long rods or line posts. There are mainly three types of insulators used for the purpose of overhead insulator. They are a. Pin Insulator, b. Suspension Insulator and c. Strain Insulator. There are two more types of electrical insulators which are available mainly for low voltage application and are called Stay Insulator and Shackle Insulator. The insulators are made of glass, polymers and porcelain. Each model is made up with different tensile strengths, densities and different levels of performance in typical working conditions. Ceramic insulators are generally used in power transmission and distribution lines for a long time. In the recent times, polymeric insulators have become widely useful due their superior insulation performance, in terms of contamination endurance compared with conventional ceramic insulators $[1,2]$.

Inspection of defects needs to be done for a wide variety of items. Generally they depend on i) Size of the item and ii) Level of details required [18]. The types of items to be inspected are: a. Large scale: sagging spans, broken or slack stay wires, leaning poles, and tree encroachment; $b$. Medium scale: equipment fixed on poles, air break switches, high and low voltage fuse units anti-climbing guards, and safety notices; c. Small scale: chipped or broken insulators, corroded joints on conductors causing discoloration, and traces of arcing on switches or fuse gear [25]. The symptoms related to each problem on overhead lines were identified and quantified in the report of EPRI [32]. Since the 1970s, the focus on cracking of insulators has been observed increasingly, because safety issues related to mechanical factors have declined [4]. Looms has briefed [52] that the damage of pin and cap disc porcelain insulators is mainly due to cement growth, cycling and corrosion. As per Cherney, porcelain suspension insulator failures are due a volume expansion of the hardened Portland cement grout in the pin hole of the insulator which in turn causes radial cracks [54] in the porcelain suspension insulators shells. When there is sufficiently low surface resistance, there will be elongation of partial discharges along the insulator profile which eventually results in flashover of insulator [51]. The emerging defects formed in the beginning stages of manufacturing process also are the causes of failure of insulator [55]. When image processing techniques are considered for automatic condition monitoring, there is an important problem of presence of background elements on the images. Usually for the images taken from the ground, the background is quite easy to remove sky with clouds, because of the high contrast. Still, there may be a situation for aerial images and those taken from the ground, where the background is much more complex like presence of trees, buildings or roads. Both cases are tackled by some authors [1]

To meet the growing demand for reliable energy supply, electric utilities are seriously motivated to drop maintenance costs by running inspection programs to locate, follow-up and repair any substantial failures at earliest possible stage [26]. Efforts on research focuses on automating the power line inspection process by looking for approaches that fulfill the various requirements of the inspection [28]. The wellbeing analysis of providing a means to perceive the likelihood of the system's approaching hazardous state indicates whether the insulator distribution system is in a good or close to a risky state [9]. Various methods 
or techniques used for monitoring the condition and classification of the insulators and the analysis done as per the literature are listed in Table 1.

Table 1. List of Defect Detection Methods

\begin{tabular}{|c|c|c|}
\hline Method & Reference & Analysis done \\
\hline $\begin{array}{l}\text { Aerial inspection } \\
\text { using manned } \\
\text { helicopter }\end{array}$ & {$[15,18]$} & Produced a theoretical and experimental basis for specifying the camera pointing accuracy \\
\hline $\begin{array}{l}\text { Modified } \\
\text { Hough transform, } \\
\text { SVM model }\end{array}$ & [36] & $\begin{array}{c}\text { Modified Hough transform and color features are extracted and SVM is used for } \\
\text { classification }\end{array}$ \\
\hline $\begin{array}{l}\text { Combined } \\
\text { Hough transform, } \\
\text { SVM model }\end{array}$ & [37] & Wavelet transform is used for feature extraction and SVM is used for classification \\
\hline $\begin{array}{l}\text { DOST-ANFIS } \\
\text { Combined approach }\end{array}$ & [1] & Discrete orthogonal S-transform is used for detection and ANFIS is used for classification \\
\hline $\begin{array}{c}\text { Combined } \\
\text { Wavelet, HMM }\end{array}$ & [34] & $\begin{array}{l}\text { Template design, feature extraction using wavelet transform and HMM for well-being } \\
\text { analysis of insulators }\end{array}$ \\
\hline $\begin{array}{l}\text { Wavelet-SVM } \\
\text { Combined Approach }\end{array}$ & & \\
\hline $\begin{array}{c}\text { DOST-SVM } \\
\text { Combined approach }\end{array}$ & [9] & Wavelet features are extracted and SVM is used for classification \\
\hline $\begin{array}{l}\text { Wiener filtering, } \\
\text { FCM,Connected }\end{array}$ & [35] & Discrete orthogonal S-transform is used for detection and SVM is used for classification \\
\hline $\begin{array}{c}\text { component labeling } \\
\text { Near-field }\end{array}$ & [33] & $\begin{array}{l}\text { Insulator image is segmented based on the improved FCM algorithm and contour of } \\
\text { insulator is labeled by using connected component labeling algorithm }\end{array}$ \\
\hline $\begin{array}{l}\text { Microwave imaging, } \\
\text { ANN }\end{array}$ & [40] & $\begin{array}{l}\text { A novel artificial neural network (ANN) - based near-field microwave nondestructive } \\
\text { testing technique for defect detection and classification in non ceramic insulators }\end{array}$ \\
\hline
\end{tabular}

The ultimate objective of the insulator detection system is to categorize defects into good or defective classes. A number of features have to be extracted from regions of interest and ideally, different combinations of these features are required to match uniquely with the features of different types of defective insulators. The various types of classification algorithms and the features extracted are shown in the Table 2. The matching is generally done using adaptive learning methods supervised or unsupervised.

Table 2. List of defect classification methods

\begin{tabular}{ccc}
\hline Paper & Method of Classification & Features extracted \\
\hline$[36]$ & SVM & RGB color features \\
{$[9]$} & SVM & Statistical features \\
{$[34]$} & HMM & Statistical features \\
{$[1]$} & ANFIS & DOST features \\
{$[35]$} & ANFIS \& & Mean, Variance \\
\hline
\end{tabular}

\section{AUTOMATIC INSPECTION SYSTEM}

The Distribution Automation System (DAS) was defined by Institute of Electrical and Electronic Engineers (IEEE) as a system which facilitates an electric utility to coordinate, monitor and operate the distribution components remotely, from remote locations in a real time mode [48]. The DAS involves collection of data and analyze the information to make, implement the control decisions and to achieve desired result [49]. The Distribution Control Centre (DCC) is the location from where control decisions are initiated. In day to day operation and maintenance of power distribution network Power Distribution Automation is a developing field and is very useful [22]. Enactment of electricity Act 2003 of India has been pressurizing the power distribution utility to make a nice plan to achieve Distribution Automation without 
further delay [47]. The automation system can be designed using the available resources like computer systems, control systems and metering systems. The tools such as computers and Remote Terminal Units (RTUs) are used for automation which uses utilities to control distribution systems and enhance efficiency, reliability, and quality of electric service [50]. The following are the key elements of the automatic inspection system of distribution system insulators.

\subsection{Image Acquisition and Processing}

Traditionally the crews are sent out by the utilities either on foot or in vehicles to drive the lines, with frequent stops to send the linemen climb the towers for closer inspection [41]. An alternative approach is to train inspectors' fly aboard the helicopters in inspecting the lines with binoculars and cameras, record the data in a log book as the helicopter hovers over and around power lines [19]. As a promising alternative, a helicopter with automatic video surveillance system was suggested [25] but difficulty comes because of rotation of the camera in its gimbals and the translational motion of the helicopter [20]. An innovative approach using RTUs as well as surface vehicular patrolling (SVP) was validated by different researchers. With the availability of both high resolution video cameras and communication networks for mass use, an arrangement of intelligent RTUs fitted with the required equipment can send the images of the insulators to the control room at regular interval for further analysis [1,35]. Since the overhead distribution networks in India mostly run parallel to the roads, SVP approach seems to be a viable alternative $[9,34,36,37]$ to the aerial inspection method using helicopters, which is not only costly and also there is possibility of danger.

\subsection{Extraction of Images Pertaining to Insulators}

Traditional approaches to automatic power line inspection [26,45] are based on human observation and can be performed simply from the ground, with climbing, using bucket truck methods or an airborne platform [46]. To reduce the inspection and maintenance costs, new approaches based on machine vision techniques with analysis of video sequences recorded during the patrol have been introduced. This resulted in increased robustness of the power system and also is helpful in the automated documentation. Usage of machine learning techniques is adaptable to the DSA and thus emerges as a viable option for real-time implementation. The insulator monitoring system described is depicted in the block diagram shown in Figure 1. The salient features are: a. image acquisition containing poles as well as insulators, $b$. extraction of image pertaining to insulators and c. condition monitoring of insulators using different machine learning techniques.

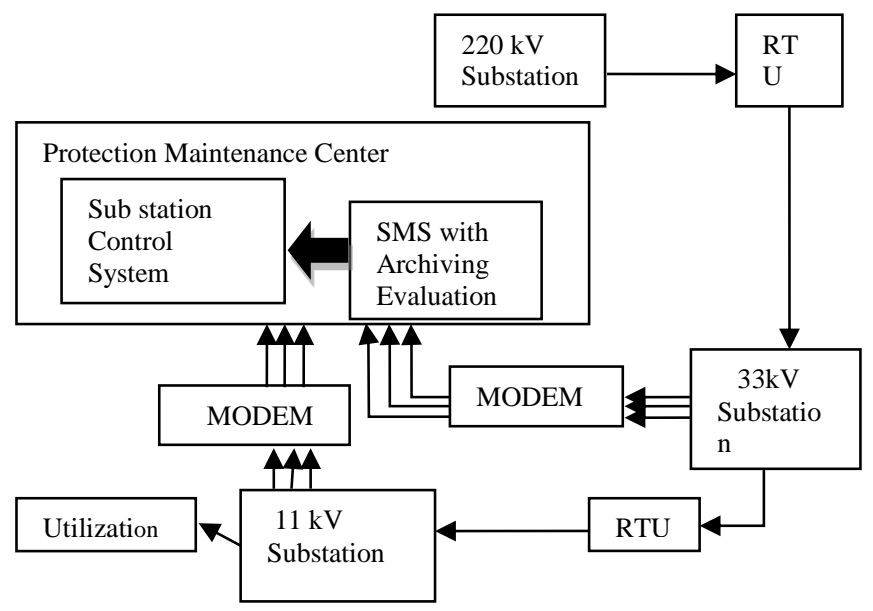

Figure 1. Insulator Monitoring System

The power distribution system crosses mountains and forests. Therefore, keeping in view all possible situations, plain back ground and complex back ground images have been considered as test cases to extract insulator images using color features and wavelet features by some authors [1, 35, 36, 37]. To simplify the task of acquiring the images containing only insulators, image segmentation has been successfully done using proven techniques such as Canny edge detection, Hough transform in conjunction with SVM [37]. In each bounding box, the insulator's presence was detected by extracting some features like color features [33] and extracted features from the bounding boxes were supplied to an SVM classifier and presence of insulator was detected. 


\section{DISCUSSION ON DEFECT DETECTION METHODS}

The insulators of a power distribution system are monitored using a defect detection and classification in three steps procedure: Segmentation localize candidate defects or regions of Interest (RoI), feature extraction from RoIs and lastly, good or defective classification. The segmentation has been very successful in $[1,9,33,34,35,36,37]$ by the use of various types of techniques. In the case of minor cracks on the insulators, the detection alone is a very useful tool to identify the defect and perfect level of classification is achieved with ease.

\subsection{Spatial Domain-based Methods}

There is plenty of literature available for automatic detection of electric towers and few references for extraction and classification of insulators. Dutta, Tanima, et al [43] have taken aerial imagery using Unmanned Aerial Vehicle (UAV) with varying natural and complex surroundings to detect line faults using a novel morphological operator, and a robust image space heuristics to locate and extract power lines completely. Martinez, et al. presents an approach [44] focusing on autonomous detection in real-time, electric towers localization and tracking using a strategy to train a two-class multilayer perceptron (MLP) neural network and applied over sliding windows for each camera frame until a tower is detected. Electric towers as well as the insulators are extracted by V.S.Murthy et al. [36, 37] by converting the colour images first into grey scaled images and then Canny edge detection which is followed by the modified Hough transform. It was used to isolate features of a particular shape within an image and segmentation is realized by applying edge detection and thresholding methods. It is also used as a tool for edge linking too. In another technique, $\mathrm{K}$-means clustering has been used to locate the proper bounding boxes containing the insulators. From the bounding boxes, features like mean, standard deviation were extracted and successfully tested for automatic insulator extraction from plain background [35] and complex background [1] as well. Fuzzy C-Means algorithm (FCM) as proposed by Bo Wen Wang, Quan Gu [33] is used to recognize transmission line insulators. To filtrate and recover image in pre-processing, the improved Wiener filter algorithm was used and then, improved FCM was used to segment the insulator. Finally, the contour of insulator is labeled by using connected component labeling algorithm. Some efficient algorithms such as template design and mean shift tracking have successfully tracked the poles in the streaming video for the subsequent process of identifying insulators [34]. Among the clustering methods used to segment the object of interest, the KMeans algorithm involves more error cluster pixels, whereas FCM algorithm involves less. The algorithm using FCM [33] have a good segmentation effect, effectively reducing the number of error cluster pixels.

\subsection{Frequency Domain-based Methods}

In the reported research on condition monitoring of the insulators, the degree of damage of an insulator affects the distribution system in different ways and so the defect of insulators have been categorized into either three states namely good, marginal and risk states or two states, good and risky. To understand the condition of insulators from the acquired images, the adopted features extracted by authors uses families of wavelet transform [34,36, 37] as well as discrete orthogonal S-transform (DOST) [1,35].

If the object's size is small or there is low contrast, generally they are to be examined at high resolutions. If their size is large or contrast is high, a coarse view is needed. If both small and large objects, as well as low and high contrast objects are simultaneously present, it is beneficial to study them at several resolutions. This concept is the fundamental motivation for multi-resolution analysis (MRA) [30]. The wavelet transform [69] has the natural ability in capturing even small cracks on the insulator using MRA along with a classifying technique like SVM to discern the health of the insulator. The DOST is used in finding the condition [56, 57] of insulator from the extracted images. It gives a spatial frequency representation like DWT. It also has an additional benefit that phase properties of the ST and FT are maintained and retains the ability to collapse exactly back to the Fourier domain [58].

\section{DEFECT CLASSIFICATION SYSTEMS}

\subsection{Discussion on Defect Classification Methods}

Once the detection of insulator is done, classification of defects in electrical power system insulators to identify and subsequently replacing the faulty insulators hence preventing heavy damages. The intelligent techniques such as SVM, HMM (Hidden Markov Model) and ANFIS (Adaptive Neuro Fuzzy Inference System) have used such features to perform the task of automated condition monitoring of the insulator efficiently. As SVM operates on structural risk minimization principle in minimizing an upper bound based on the generalization error, and deals with only two parameters, for classification and it has been chosen for effective, faster insulator condition monitoring for automation purposes by the researchers [1], [9]. ANFIS is an adaptive network which is similar to adaptive network simulator of fuzzy controllers and it is equivalent to 
a FIS. Back propagation gradient descent and least square method for non-linear and linear parameters is used to adjust the parameters for a given input or output data set [8]. A comprehensive review of common machine learning techniques like ANN, SVM, and GMM along with HMM that are used in automatic speech recognition is given in [61].

\section{2. $\quad$ Support Vector Machines (SVM)}

SVM is a set of supervised learning algorithm which can be used for classification and regression [64]. It is found as a very effective technique for general purpose supervised pattern recognition as proposed by Vapnik et al. [67]. It is a binary non-linear classifier employed to predict whether an input value belongs a class 1 or a class 2 and is generally used to classify an object into a defective or good one. To classify the insulators, SVMs can be used to separate the given set of labeled data with a hyper plane with the maximum margin. As most of the practical classification problems are non-linear, the SVMs use kernel functions that automatically realize a non-linear mapping to a feature space. Shape recognition of tyre marking points [65] is a recent application for SVM.

SVM is also extended to solve multiclass separation problem which uses one-versus-all and oneversus-one techniques. Multiclass problem solution using SVM has been reported in [35, 36, 37]. A few binary classifiers are needed to be trained to for a multiclass classification problem. The performance of SVM classification is strongly related to the choice of the kernel function. There are many number of kernel functions available as follows: linear kernel function, radial basis function, polynomial kernel function et al. Among them, Radial Basis Function (RBF) is the most popular one. The samples are non-linearly mapped into a higher dimensional space. The linear kernel is a special case of RBF [68]. The features extracted from DOST, along with SVM and ANFIS [1] were used to estimate the condition of the insulator. In the referred papers [1,35] SVM is used for two purposes, i.e. for locating the proper bounding boxes containing the insulators amongst the bounding boxes using features (like mean and standard deviation) extracted from the cropped images after applying some segmentation technique and also for classifying insulator according to its condition. So, the output of first SVM gives bounding boxes having insulators and second SVM discriminates, whether the insulator is healthy or broken.

\subsection{Hidden Markov Model (HMM)}

HMM is a statistical Markov model consisting of a Markov chain with finite number of states, a state transition probability matrix, and an initial state probability distribution. Although the states are unobservable, but the output which is dependent on the state, is visible and are drawn as per a probability distribution [59]. HMMs were used for face detection and recognition and they were motivated because they are partially invariant to variations in scaling and also the structure of images [60-62], [72]. Application of HMMs is done in many fields where the goal is to recover a data sequence that is not observable immediately whereas some other data are available that depend on the sequence. It is being used in several fields including facial expression recognition (FER) [63] with improved accuracy than existing methods. An algorithm given in [34] utilizes hidden Markov models to determine the health condition of insulators. Since insulators on poles possess a lot of variations in terms of features, and also requires scaling, HMM has been employed by those authors. HMM has been used for well-being analysis to segregate a good insulator from a bad one using Baum-Welch algorithm.

\subsection{Adaptive Neuro-fuzzy Inference System (ANFIS)}

For a given input or output data set, the ANFIS adjusts all the required parameters. It uses a method of back propagation gradient descent for non-linear parameters and least square type of method for linear parameters [70]. The neuro-adaptive learning techniques provide a methodology for the fuzzy modeling procedure in extracting information about a data set. This in turn is used to compute the parameters required for membership functions that needed for the FIS to rightly correlate input or output data in fuzzy domain.

\subsection{Comparative Analysis}

The comparative study done by Murthy et al. reported that $92 \%$ success rate was achieved using HMM with wavelet features [34] and established that this approach performs more efficiently in comparison to SVM with color features [36] and SVM with wavelet MRA features [9]. The classification accuracies reported by recent literature are presented in the Table 5. Due to its accuracy of insulator damage detection, the HMM leads to quicker maintenance and restoration of power supply compared to the use of SVM [36, 37]. It is also shown that FIS, with its inherent ability, computes the health of insulators in terms of the degree to which the insulators are in good, marginal or risky states. A comparison between the methods, DOST-ANFIS and DOST-SVM was done by Reddy et al. [1] incorporating the complex background images 
and results prove the effectiveness of the proposed techniques in dealing with different possibilities of complex backgrounds. They would otherwise lead to wrong conclusions about the condition of insulators.

Table 5 Comparison of Feature Extraction and Defect Classification Techniques

\begin{tabular}{cccc}
\hline $\begin{array}{c}\text { Classification } \\
\text { Approach }\end{array}$ & $\begin{array}{c}\text { Feature } \\
\text { vector } \\
\text { type }\end{array}$ & $\begin{array}{c}\text { Classification } \\
\text { Accuracy (\%) }\end{array}$ & $\begin{array}{c}\text { Reference } \\
\text { No. }\end{array}$ \\
\hline SVM & Colour & 85 & {$[36]$} \\
SVM & Wavelet & 90 & {$[9]$} \\
HMM & Wavelet & 92 & {$[34]$} \\
ANFIS & DOST & - & {$[35]$} \\
SVM \& ANFIS & DOST & - & {$[1]$} \\
\hline
\end{tabular}

\section{SCOPE FOR FUTURE WORK}

There are several tools both in the spatial and frequency domains like local binary pattern (LBP), curvelet transform, and contourlet transform etc., for feature extraction. Deep belief networks (DBNs) which is a representative method of deep learning [71] and Extreme Learning Machine (ELM) can be used for classification. The newly emerged machine learning theory, deep learning may be applied for better classification which outperformed the other state-of-the-art classification methods. The various techniques proposed by the different authors did not consider any of the non-visual defects such as internal cracks, high partial discharge activities and high leakage current. Thermal cameras would be a viable option to take these aspects into consideration. The geographical inference system (GIS) may be used to obtain spatial coordinates for distribution system planning and automation. Once the images sent by the RTUs are obtained, the GIS-aided insulator monitoring would reduce the existing problems by assigning unique identification numbers to RTUs for subsequent image processing and proper monitoring of the insulators.

\section{REFERENCES}

[1] M. Jaya Bharata Reddy, Karthik Chandra B and D. K. Mohanta, "Condition Monitoring of $11 \mathrm{kV}$ Distribution System Insulators Incorporating Complex Imagery Using Combined DOST-SVM Approach", IEEE Transactions on Dielectrics and Electrical Insulation, Vol.20, Issue 2 pp. 664-674, April 2013.

[2] Dabo Zhang, Wenyuan Li, Fellow, IEEE, and Xiao fu Xiong, "Overhead Line Preventive Maintenance Strategy Based on Condition Monitoring and System Reliability Assessment”, IEEE Trans on Power Systems, Vol.29, NO.4, July 20141839.

[3] C. J. Kim, Jeong Hoon Shin, Myeong-Ho Yo GiWon Lee, "A Study on the Characterization of the Incipient Failure Behavior of Insulators in Power Distribution Line", "IEEE Transactions on Power Delivery", Vol. 14, No. 2, April 1999 pp 519-524.

[4] R.K. Aggarwal, A.T. Johns, J.A.S.B. Jayasinghe, "An overview of the condition monitoring of overhead lines", Electric Power Systems Research 53 (2000) 15-22, , W. Su, pp.15-22

[5] H. H. Kordkheili, H. Abravesh, M. Tabasi, M. Dakhem, and M. M. Abravesh, "Determining the probability of flashover occurrence in composite insulators by using leakage current harmonic components," IEEE Trans. Dielectr. Electr. Insul.,vol.17, no.2, pp.502-512, 2010.

[6] C. Andrea, et al, "Inferring ceramic insulator pollution by an innovative approach resorting to PD detection," IEEE Trans. Dielectr. Electr. Insul., vol. 14, no. 1, pp. 23-29, 2007

[7] S.Venkataraman, et al, "Impact of weathering on flashover performance of non ceramic insulators," IEEE Trans. Dielectic. Electr. Insul., vol.15, no.4, pp.1073-1080,2008.

[8] I. Ramirez-Vazquez, R. Hernandez-Corona, and G. Montoya-Tena, "Diagnostics for nonceramic insulators in harsh environments, "IEEE Electr. Insul. Mag., vol. 25, no. 6, pp. 28-33, 2009.

[9] V. S. Murthy, et al, "Insulator condition analysis for overhead distribution lines using combined wavelet support vector machine, "IEEE Trans. Dielectr .Electr. Insul., vol. 17, no. 1, pp. 89-99, 2010.

[10] M.Komoda, et al , "Electromagnetic induction method for detecting and locating flaws on overhead transmission lines," IEEE Trans. Power Del., vol.5, no.3, pp.1484-490,1990.

[11] H.Zangl et al, "A feasibility study on autonomous online condition monitoring of high-voltage overhead power lines,” IEEE Trans. Instrum. Meas., vol. 58, no. 5, pp. 1789-1796, 2009.

[12] T. Hjartarson, et al, "Development of health indices for asset condition assessment," in Proc. IEEE Trans. and Dist. Conf. Expo., 2003, vol.2, pp.541-44.

[13] Y. Han and Y. H. Song, "Condition Monitoring Techniques for Electrical Equipment-A Literature Survey", IEEE transactions on power delivery, vol. 18, no. 1, pp. 4-13, Jan. 2003

[14] D. I. Jones, C. C. Whitworth, G. K. Earp and A. W. G. Duller, A laboratory test-bed for an automated power line inspection system, Control Engineering Practice, Vol. 13, No.7, pp. 835-851, 2005.

[15] D. I. Jones and G. K. Earp, Camera sightline pointing requirements for aerial inspection of overhead power lines, Electric Power Systems Research, Vol. 57, pp. 73-82, 2001. 
[16] C. C. Whitworth, et al, "Aerial video inspection of power lines", Power Engineering J., Vol.15, No.1, pp.25-32, 2001.

[17] Ian Golightly and Dewi Jones, "Corner detection and matching for visual tracking during power line inspection," Image and Vision Computing, vol. 21, pp. 827-840, 2003.

[18] A. W. G Duller C. C. Whitworth, D. I. Jones and G. K. Earp, "Aerial video inspection of overhead power lines," IEE Power Engineering Journal, vol. 15, no. 1, pp. 25-32, 2001.

[19] Aerospect, "Utah state to revolutionize power line inspections," http://www.spacedaily. com/news/energy -tech03ze.html, 2003.

[20] D. I. Jones, "Aerial inspection of overhead power lines using video: Estimation of image blurring due to vehicle and camera motion," in Proc IEE Vision, Image and Signal Processing, 2000, pp. 157-166.

[21] F. Xu, et al, "Pedestrian detection and tracking with night vision", IEEE Trans. Intelligent Transportation Syst., Vol. 6, pp. 63-71, 2005.

[22] R. P. Gupta, et al., "Automated Verses Conventional Distribution System", Proc. of the Third International Conference on Power and Energy Systems EuroPES-2003, Spain, 2003, pp. 33-38.

[23] A. Pahwa and J. K. Shultis, Assessment of the Present Status of Distribution Automation, Engg. Experiment Station, Kansas State niv., Manhattan, KS, Rep. 238, 12.

[24] R. P. Gupta and R. K. Verma, Power System Automation :Academic open Internet journal, Volume 15, 2005, Department of Electrical and Computer Engineering University of Western Ontario.

[25] Lili Ma, Yang Quan Chen: Aerial Surveillance System for overhead power line inspection, Technical report USUCSOIS-TR-04-08 (2004), Utah State University, USA.

[26] B. Avidar: Electronic airborne inspection methods for overhead transmission power-lines. 6th Int. Conf. on Transmission and Distribution Construction and Live Line Maintenance, p. 89-93, 1993.

[27] J. Katrasnik, et al "A survey of mobile robots for distribution power line inspection", IEEE Transactions on Power Delivery, 25(1), 2010.

[28] Sampedro, Carlos, et al. "A supervised approach to electric tower detection and classification for power line inspection." Neural Networks (IJCNN), 2014 International Joint Conference on. IEEE, 2014.

[29] Pan, Lifeng, "Intelligent Image Recognition Research on Status of Power Transmission Lines," Sensors \& Transducers (1726-5479)179.9 2014.

[30] R.C. Gonzalez, R.E. Woods, Digital Image Processing, 3rd edn. (Pearson Education, 2008). ISBN 978-81-3171934-3.

[31] S. Haykins, Neural Networks, 2nd edn.(Pearson Education, 1999). ISBN 81-7808--300-0

[32] Investigation of applying new technologies to overhead transmission line inspections, Project 1497-2, Electric Power Research Institute Report, September 1981.

[33] Bo Wen Wang, Quan Gu, A Detection Method for Transmission Line Insulators Based on an Improved FCM Algorithm, TELKOMNIKA, Vol.13, No.1, March 2015, pp. 164 172

[34] Velaga Sreerama Murthy, et al, "Digital Image Processing approach using combined Wavelet- Hidden Markov Model (HMM) for well-being analysis of insulators", International Journal of IET image processing, Vol.5, Iss.2, 2011, pp 171-183

[35] M. Jaya Bharata Reddy, B. Karthik Chandra, D. K. Mohanta, "A DOST Based Approach for the Condition Monitoring of $11 \mathrm{kV}$ Distribution Line Insulators", IEEE Trans. on Dielectrics and Electrical Insulation, Vol. 18, Issue 2, pp. 588-595, April 2011.

[36] V.S. Murthy, et al , Distribution system insulator monitoring using video surveillance and SVM for complex background images, Int. Journal of Power and energy conversion, Vol.1, No.1, pp 49-72, 2009

[37] S. Murthy, D. Mohanta, S. Gupta, Distribution system insulator monitoring using video surveillance and SVM for complex background images, Int. Jnl. of Comp Appl and Tech., Vol 3, No.1, pp 11-31, 2011

[38] Zhu, Hu, W. G. Li, and Ye Lin. "Present and future development of detection methods for composite insulator." Insulators and Surge Arresters 8.1 (2006): 133-137.

[39] T Guo, W Wang, H Yang, X Yuan"Research on live line auto-testing technology for transmission line insulators." (2015), 3rd International Conference on Mechatronics, Robotics and Automation.

[40] Qaddoumi, et al, "Outdoor Insulators Testing Using Artificial Neural Network-Based Near-Field Microwave Technique." Instrumentation and Measurement, IEEE Trans on 63.2 (2014): 260-266.

[41] Palo ALto, "EPRI aerial inspection system all set for takeoff," http://www.epri.com/ corporate/discover, epri/news/ 2001releases/ 011017 aerial.html, 2001.

[42] R. P. Gupta, and S. C. Srivastava, "Technology development and implementation for power distribution automation”, Water and Energy International J., Vol. 61, pp. 40-47, 2004.

[43] Dutta, Tanima, et al. "Image Analysis-Based Automatic Detection of Transmission Towers using Aerial Imagery." Pattern Recognition and Image Analysis.Springer International Publishing, 2015.641-651.

[44] Martinez et al, Towards autonomous and tracking of electric towers for aerial power line inspection, Unmanned Aircraft Systems (ICUAS), 2014 International Conference on

[45] A. Philips et al: Airborne Inspection Technology: Market Survey. Technical Report 1006749 , EPRI, Palo Alto, California , USA 2002.

[46] Mazurek, et al. "Application of background estimation and removal techniques for the extraction of the power line components on the digital images for the automatic power line inspection systems."Pomiary, Automatyka, Kontrola 54 (2008): 698-699. 
[47] Lahiri, et al. "Importance of distribution automation system for Indian power utility." Power \& Energy Soc. Gen. Meeting2009 PES'09 IEEE.

[48] D. Bassett, K. Clinard, J. Grainger, S. Purucker, and D. Ward, “Tutorial Course: Distribution Automation”, IEEE Tutorial Publication 88EH0280-8-PWR, 1988.

[49] J. B. Bunch, "Guidelines for Evaluating Distribution Automation", EPRI Report EL-3728, 1984.

[50] S.S. Venkata, et al. , "What future distribution engineers need to learn", IEEE Trans. Power Syst., Vol. 19, pp. 1723, 2004.

[51] Chandrasekar, S., et al. "Investigations on leakage current and phase angle characteristics of porcelain and polymeric insulator under contaminated conditions." Dielectrics and Electrical Insulation, IEEE Transactions on 16.2 (2009): 574-583.

[52] J.S.T. Looms, Insulators for High Voltages, Peter Peregrinus Ltd, 1988.

[53] E.A. Cherney, Cement growth failure of porcelain suspension insulators, IEEE Trans. PAS-102 (8) (1983) 27652774.

[54] E.A. Cherney, Cement growth failure mechanism in porcelain suspension insulators, IEEE Trans. PWRD-2 (1) (1987) 249-255.

[55] A.L. Barclay, D.A. Swift, Cap and pin insulators: electrical puncture of porcelain under AC energisation, $5^{\text {th }}$ International Conf on Dielectric Materials, Measurements and Applications, 1988, pp. 370-374

[56] R.G. Stockwell, "A basis for efficient representation of the S-transform", J. Digital Signal Processing, Vol.17, pp 371-393, 2007.

[57] S. Drabycz, R. G. Stockwell and J. R. Mitchell, "Image texture characterization using the discrete orthogonal stransform”, J. Digital Imaging, Vol. 22, pp. 696-708, 2009.

[58] Y. Wang and J. Orchard, "The discrete orthonormal Stockwell transform for image restoration", 16th IEEE Intern. Conf. Image Processing (ICIP), pp. 2761 - 2764, (DOI: 10.1109/ICIP.2009.5414135), 2009.

[59] L. R. Rabiner, "A Tutorial on Hidden Markov Models and Selected Applications in Speech Recognition", Proceedings of the IEEE, Vol. 77, No. 2, pp. 267-295, 1989.

[60] S. Eickeler, et al, "Hidden Markov Model Based Continuous Online Gesture Recognition", $14^{\text {th }}$ Int. Conf. on Pattern Recognition, Vol. 2, pp.1206-1208, 1998.

[61] Jayashree Padmanabhan, Melvin Jose Johnson Premkumar, Machine Learning in Automatic Speech Recognition: A Survey, IETE Technical Review, Vol. 32, Iss. 4, pp. 240-251, July-Aug. 2015

[62] A. V. Nefian and M. H. Hayes, "Face Detection and Recognition Using Hidden Markov Models", Int. Conf. on Image Processing, Chicago, Vol. 1, pp. 141-145, 1998.

[63] Muhammad Hameed Siddiqi, et al. Depth Camera-Based Facial Expression Recognition System Using Multilayer Scheme, IETE Technical Review, Vol. 31, Iss. 4, pp. 277-286, Sept-Oct. 2015.

[64] C. Burges, "A Tutorial on Support Vector Machines for Pattern Recognition" In U. Fayyad, editor, Proceedings of Data Mining and Knowledge Discovery, pp. 1-43, 1998.

[65] Yong Wang, Hui Guo, "Shape Recognition of Tyre Marking Points Based on Support Vector Machine", IETE Technical Review, Vol. 32, Iss. 2, pp.123-130, Mar-Apr. 2015.

[66] T. Kanungo, D.M. Mount, N. S. Netanyahu, C. D. Piatko, R. Silverman and A. Y. Wu, "An efficient k-means clustering algorithm: Analysis and implementation", IEEE Trans. Pattern Analysis and Machine Intelligence, Vol. 24, pp 881-892, 2002.

[67] Cortes, C. and Vapnik, V. (1995) 'Support vector networks', Machine Learning, Vol. 20, pp.1-25.

[68] S. S. Keerthi and C. J. Lin. "Asymptotic behaviors of support vector machines with Gaussian kernel", Neural Computation, Vol. 15, pp. 1667-1689, 2003.

[69] S. G. Mallat, "A Theory for multi resolution signal decomposition: The wavelet representation", IEEE Trans. Pattern Analysis Machine Intelligence, Vol. 11, pp. 674-693, 1989.

[70] M.J.B. Reddy, D.K. Mohanta, "Adaptive-neuro-fuzzy inference system approach for transmission line fault classification and location incorporating effects of power swings", IET Generation, Transmission, Distribution, Vol. 2, pp. 235-244, 2008.

[71] Xiaoming Zhao, Xugan Shi, Shiqing Zhang, "Facial Expression Recognition via Deep Learning”, IETE Technical Review, Vol. 32 Vol. 32, Iss. 5, 2015.

[72] A. V. Nefian and M. H. Hayes, "A Hidden Markov Model-Based Approach for Face Recognition", Proceedings of IEEE Int. Conf. on Acoustic, Speech and Signal Processing, Vol. 5, pp. 2721-2724, 1998. 